\title{
Power Maximization using Multiple Step, Load-Side, Current-mode Sensing
}

\author{
Naseem Ramli*, Stuart Walker \\ School of Computer Science and Electronic Engineering CSEE \\ University of Essex \\ Colchester, UK \\ nramlia@essex.ac.uk / stuwal@essex.ac.uk
}

\begin{abstract}
This paper presents a novel load-side maximum power point tracker using a multiple step difference algorithm. This technique maximizes the power into any given load using a current-mode, load-side controller under various insolation levels. MATLAB/Simulink was used for simulation studies using a normalized, heuristic, photovoltaic model while an off-theshelf, four-switch buck-boost converter was employed along with a controllable, indoor, built-in-house, solar simulator for experimental validations. The proposed method guarantees maximum power tracking under various weather conditions and operates at unity power factor on a self-synchronized basis.
\end{abstract}

Keywords; Photovoltaic PV; Maximum power point tracking MPPT; Forward-backward algorithm; Load-side optimizer, single and multiple -step difference

\section{INTRODUCTION}

Photovoltaic (PV) power systems are currently considered as an important alternative power source due to the ready availability of high-efficiency PV panels and concerns about environmental issues. Solar power is at the forefront of the alternative domestic power generation due to the large amount of power in incident sunlight in both open fields and residential sites. Of course, partial obstruction by trees and large building shadows conspire to limit the availability and efficiency of solar power particularly in urban and industrial situations. Thus, tracking the maximum power point of a photovoltaic module is an important part of any power generation system eliminating as it does any mismatch between solar panels and the load. Maximum power point tracking has attracted increasing attention in research over the last several years. There are a number of MPPT methods with different complexity, speed, cost and number of required sensors [1-6].

The most significant conventional MPPT algorithms and related artificial intelligent techniques have been well documented in literature, examples being: perturbation and observation ( $\mathrm{P} \& \mathrm{O})$, hill climbing, short-circuit current, open circuit voltage, incremental conductance, fuzzy logic and neural networks [7-11]. Using the technique of short-circuits current and open circuit voltage $[1,2]$ to acquire the maximum power is effective. However, the PV array must be short/opencircuited periodically to measure the short circuit current or open circuit voltage. This results in efficiency reductions and losses. For the simplicity and ease of implementation, hill climbing and $\mathrm{P} \& \mathrm{O}$ methods are widely used in PV generation systems [9]. The hill climbing method requires a perturbation in the duty cycle of the power converter, whereas the P\&O method involves perturbation in the operating voltage of the array [8]. In the case of hill climbing, perturbing the duty cycle of power converter leads to PV current perturbation and in turn perturbs the PV array voltage. Fig. 1 shows that the power increases on the left of MPP as the voltage increments whereas it decrements on the right of MPP as long as the voltage decreased. The perturbation should be kept the same to reach the MPP if there is an increase in PV output power whereas if there is a decrement in the PV output power the perturbation direction should be reversed [8].

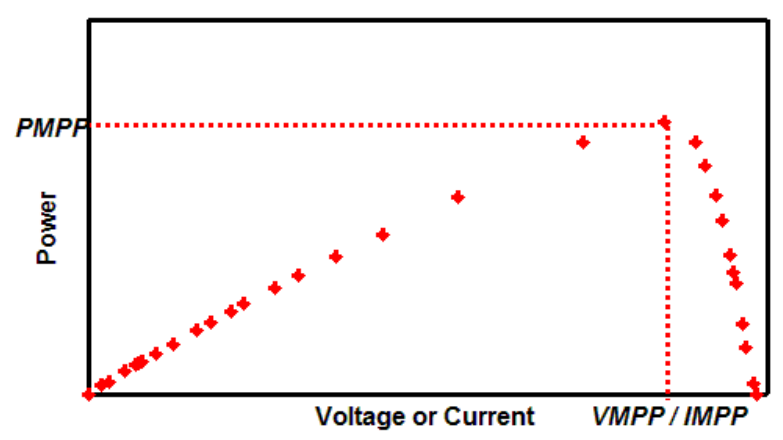

Figure 1 P-I \& P-V Curve of solar cell

Although the P\&O method is at forefront of the available methods due to its intuitive nature and implementation simplicity, it suffers from a performance unbalance between the steady state performance and the transient rise time. It has also shown that $\mathrm{P} \& \mathrm{O}$ algorithm fails to track the maximum power point under variation in insolation level [8]. Consequently, a centered differentiation algorithm can be applied to overcome the significant issues of the popular algorithms cited in literature. Less attention has been paid to this algorithm on literature. In [12], a work on centered differentiation and steepest descent to maximum power point tracking is described. However, the control system was based on complex voltage mode controller and sensing the output on the PV side.

Naseem Ramli is sponsored by Ministry of Higher Education and Scientific Research, Libya 
Generally, most recent applications of MPPT are based on the (PV side) using power converters with no attention to load protection. A lossy power converter stage between the supply side and load side causes a failure in delivering the maximum power to the load. In fact, the load must be chosen correctly to absorb all the delivered power from the PV source. However, the characteristics of a load in some applications are continuously varying. This necessitates applying the MPPT at the load side to overcome all the significant issues of over current or over voltage at any given load. This technique further enhances the tracking procedure because the optimum power delivered from PV source through the power converter the intermediate stage to the load will be guaranteed. An attempt at load side optimization has been described using a conventional $\mathrm{P} \& \mathrm{O}$ algorithm, which was compromised by varying irradiance levels [13].

This paper addresses load-side maximum power point tracking using single and multiple step difference forwardbackward algorithms. The main difference between the proposed control system and the existing one is that, instead of tracking the current or voltage output of the PV panel, the current of an intermediate stage (buck-boost converter) is maximized at any given load. To our knowledge, single and multiple steps, forward backward difference load side optimizers using current mode control have not been reported in literature. This work describes the simulation and experimental results in rapidly varying insolation conditions.

\section{PV INVERTER MODEL}

It is necessary to model the PV panel in order to simulate the proposed MPPT algorithm. The switching dynamic of the power stage conversion (DC-DC converter) is discussed in details in the following sections.

\section{A. PV Heuristic Model}

The P-R curve is modeled by a heuristic equation, which is defined as (1).

$$
\begin{array}{r}
P\left(\frac{R}{R_{\max }}\right)=\frac{2 P_{\text {max }}\left(\frac{R}{R_{\max }}\right)}{\left[1+\left(\frac{R}{R_{\max }}\right)^{2}\right]} \\
P(x)=\frac{2 x}{1+x^{2}}
\end{array}
$$

Where: $\mathrm{R}$ is the load, $\mathrm{R}_{\max }$ is the optimum load, and $\mathrm{P}_{\max }$ is the maximum power. The non-linear equation shown in (1) represents load $\mathrm{R}$ for quasi- short circuit current $1 / \mathrm{R}$ quasi open circuit voltage. Equation (2) shows the normalized version of (1), where $P_{\max }=1$ and $R / R_{\max }=x$. The waveform characteristic of a sinusoidal input is illustrated in Fig.2. It is evident that the slope of the heuristic equation is positive at
$\mathrm{R} / \mathrm{R}_{\max }>1$, while a negative slope at $\mathrm{R} / \mathrm{R}_{\max }<1$, and no slope at $\mathrm{R}=\mathrm{R}_{\text {max }}$.
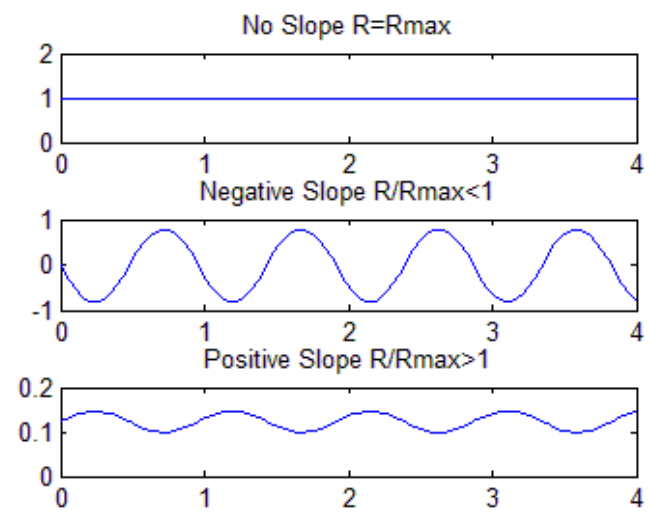

Figure 2 Relation between $P(\mathrm{x})$ and $\mathrm{x}$ in heuristic model at different $\mathrm{R} / \mathrm{R}_{\max }$

\section{B. Load Matching and Buck-Boost Converter}

The PV module cannot transfer maximum power to the load itself due to an impedance mismatch. A DC-to-DC converter is utilized to match the PV module internal impedance to any given load and adjust the operating condition to reach the maximum power point. To maximize the power into any given load above or below the supply source, a buck-boost converter is required.

At a steady state and assuming the buck-boost converter is operating in continuous mode, the relationship of the current and voltage at the load terminals with those at the PV system given by (3) and (4).

$$
\begin{aligned}
& V_{o}=\frac{D}{1-D} V_{i} \\
& I_{o}=\frac{D}{1-D} I_{i}
\end{aligned}
$$

Where: D the converter duty cycle $I_{i}$ the PV current, $I_{o}$ the load current; $\mathrm{V}_{\mathrm{o}}$ the load voltage; $\mathrm{V}_{\mathrm{i}}$ the PV voltage. Equation (3) and (4) yield:

$$
\begin{gathered}
R_{L}=\frac{D^{2}}{(1-D)^{2}} R_{i} \\
R_{i}=\frac{(1-D)^{2}}{D^{2}} R_{L}
\end{gathered}
$$

Where: $R_{L}$ is the load impedance, and $R_{i}$ is the equivalent impedance seen by the PV panel.

It is clear from (6) that, for certain load impedance, the equivalent impedance depends only on the duty cycle of the 
buck boost converter. Thus, to maximize the power into a load the duty cycle is adjusted.

\section{Prosposed Maximum Power PoInT TRACKING}

Many MPPT studies have focused on improving the tracking algorithm either for steady state performance or response speed. Developing new MPPT control techniques has received less attention. The most well-known control techniques cited in the literature are the voltage control method where the control variable is voltage reference and the duty cycle adjustment technique at which the duty cycle of the DC-to-DC converter is altered till the MPP is reached. Both control techniques are based on input sensing (PV side sensing) as illustrated in Fig.3. To protect the load from over voltage or current a load side current controller along with single and multiple-step forward backward difference algorithm is proposed in this paper.

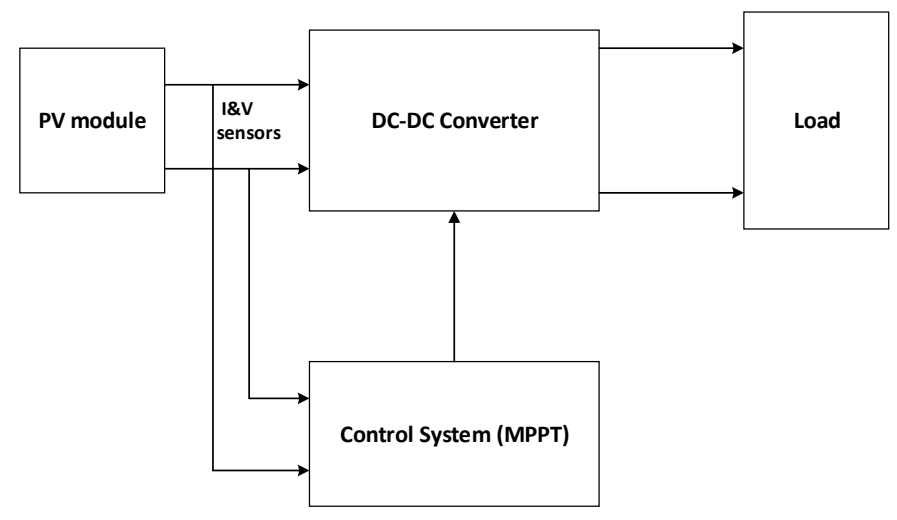

Figure 3 Block diagram of conventional MPPT

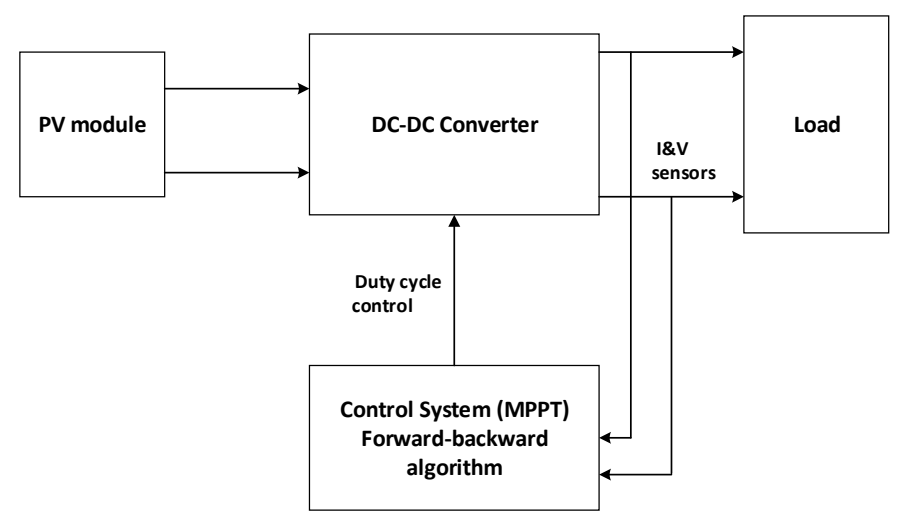

Figure 4 Block diagram of proposed MPPT

The main difference between the proposed control and existing ones is that the power is maximized at the load, instead of at the input of the power conversion. The proposed MPPT algorithm is based on first and second order central difference methods that track the maximum power at rapidly varying irradiance conditions.

\section{A. Single and Multiple Forward/Backward Difference Algorithm}

First-order, forward/backward central difference algorithms (single step forward backward) require three point measurements: $\left(\mathrm{R}_{\mathrm{i}-1}, \mathrm{P}_{\mathrm{i}-1}\right),\left(\mathrm{R}_{\mathrm{i}}, \mathrm{P}_{\mathrm{i}}\right)$, and $\left(\mathrm{R}_{\mathrm{i}+1}, \mathrm{P}_{\mathrm{i}+1}\right)$ to find the MPP. Where: $P_{i}, P_{i-1}$, and $P_{i+1}$ symbolize the sequence of $P V$ power, and $R_{i}, R_{i-1}$, and $R_{i+1}$ represent the equivalent impedances seen by the PV panel. It was demonstrated above that the slope of P-R approximation curve determined by (1) is positive when $R / R_{\max }>1$, negative slope when $R / R_{\max }<1$ no slope when $\mathrm{R}=\mathrm{R}_{\max }$.

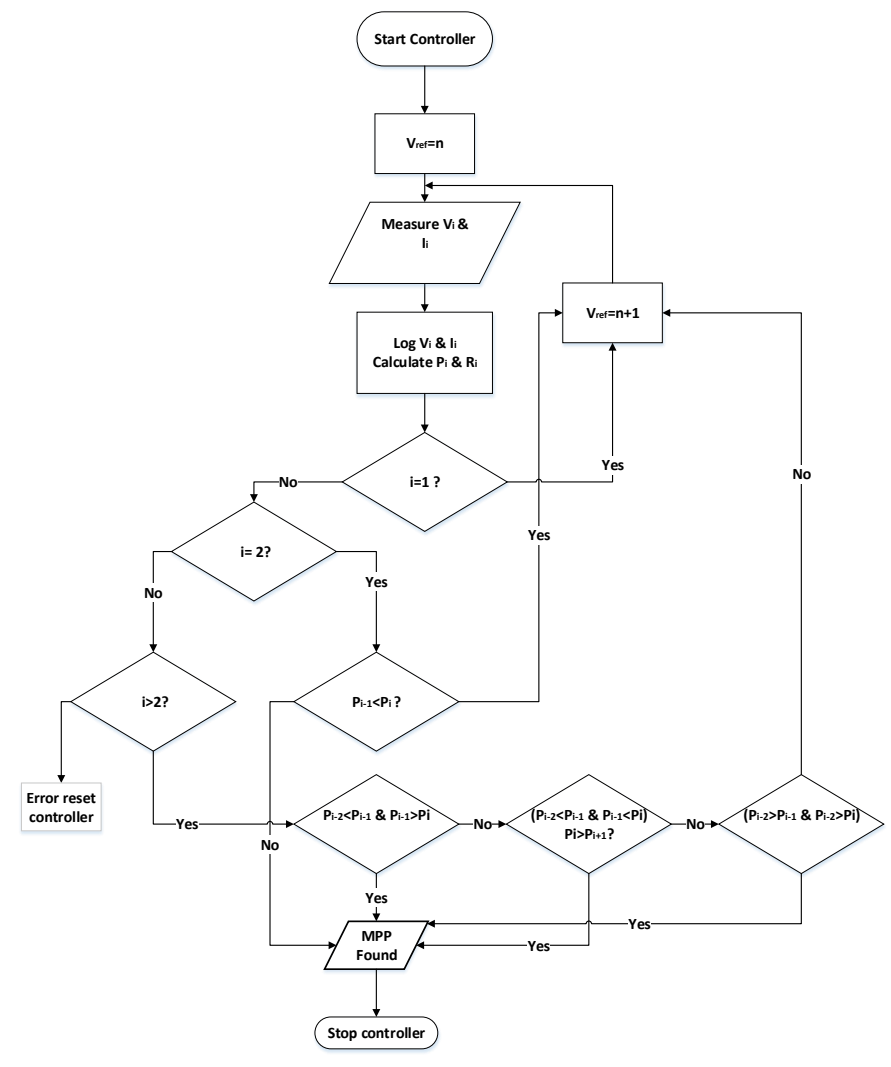

Figure 5 Proposed MPPT flowchart (First - order central difference)

The general central-differentiation equation illustrated by (7) and (8):

$$
\frac{d P}{d R}=f^{\prime}\left(R_{i}, P_{i}\right)
$$

$$
f^{\prime}\left(R_{i}, P_{i}\right) \approx \frac{P_{(i+1)}-P_{(i-1)}}{2 \Delta R}+O\left(\Delta R^{2}\right)
$$


Where: $2 \Delta \mathrm{R}$ is the distance between the forward and backward points; $\Delta \mathrm{R}$ is the incremental step of the equivalent impedance seen by the PV (altering this by the inverter duty cycle) and $\mathrm{O}\left(\Delta \mathrm{R}^{2}\right)$ is the truncated error of the relevant Taylor approximation.

$f^{\prime}\left(R_{i}, P_{i}\right)$ is zero at the MPP when $P_{i+1}-P_{\mathrm{i}-1}=0$. In fact, the maximum power is located at, $\left(\mathrm{R}_{\mathrm{i}}, \mathrm{P}_{\mathrm{i}}\right)$ which is the center between $\left(\mathrm{R}_{\mathrm{i}-1}, \mathrm{P}_{\mathrm{i}-1}\right)$ and $\left(\mathrm{R}_{\mathrm{i}+1}, \mathrm{P}_{\mathrm{i}+1}\right)$. The controller stops tracking once the MPP is located and thus reduces the ripples in the output power compared to continuous $\mathrm{P} \& \mathrm{O}$ tracking. Equation (9) represents a second order difference method.

$$
f^{\prime \prime}\left(R_{i}, P_{i}\right) \approx \frac{P_{(i+1)}-2 P_{i}+P_{(i-1)}}{(\Delta R)^{2}}+O\left(\Delta R^{2}\right)
$$

These algorithms were tested on various insolation levels using an off-the-shelf four-switch buck-boost converter that operates in current mode.

\section{B. Proposed Control System}

The new MPPT algorithm is based on first and second order central differentiation method. Figure 6 shows the block diagram of the algorithm. The algorithm works by adding a $2 \mathrm{~Hz}$ periodic sinusoidal perturbation signal $\beta \sin (\omega t)$ to $\tilde{x}$ the best estimate of $x$ that maximizes the output power. The output of this passes through the normalized heuristic PV model to produce perturbations in the output power. A highpass filter at $2 \mathrm{~Hz}$ cut off frequency approximates the DC component of $y$ which is removed by applying a third order Butterworth low-pass filter (Sallen and Key design) of 10Hz. The cascaded high-pass and low-pass filters creates a low-Q, band-pass filter with a band range from $2 \mathrm{~Hz}$ to $10 \mathrm{~Hz}$. The product of $\beta \sin (\omega t)$ and $h$ creates approximately two sinusoidal signals $n$. Integrating this signal (the gradient) and feeding it back to the system causes the control variable to track the MPP.

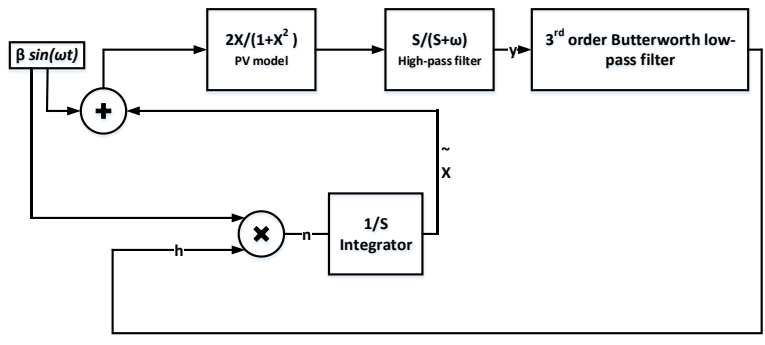

Figure 6 Proposed MPPT Algorithm

\section{MPPT RESULTS AND DISCUSSIONS}

\section{A. Simulation of Proposed MPPT (MATLAB/Simulink)}

This section summarizes the simulated results of the proposed MPPT under rapidly varying irradiance conditions. The algorithm is tested under different insolation: more irradiance level, low insolation level and reference level condition $\eta$. The reference condition is shown in Fig. 7.

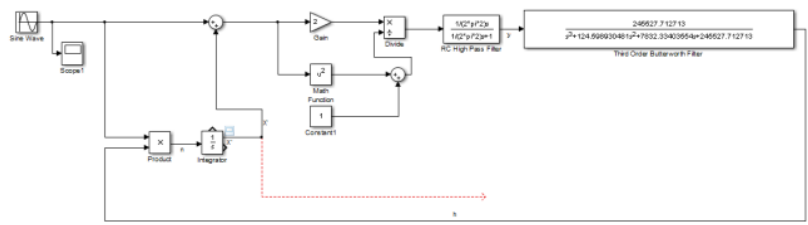

Figure 7 MATLAB/Simulink proposed MPPT implementation
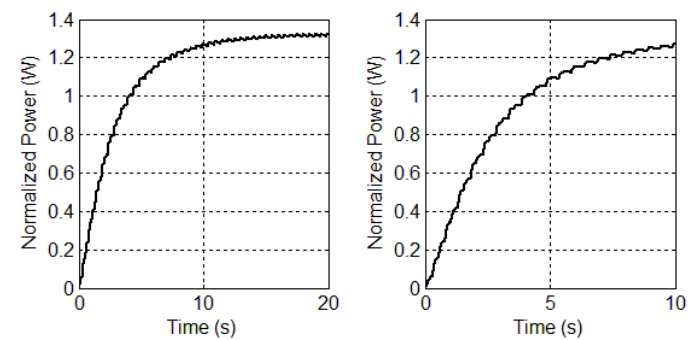

Figure 8 MPPT at the reference insolation level $\eta$
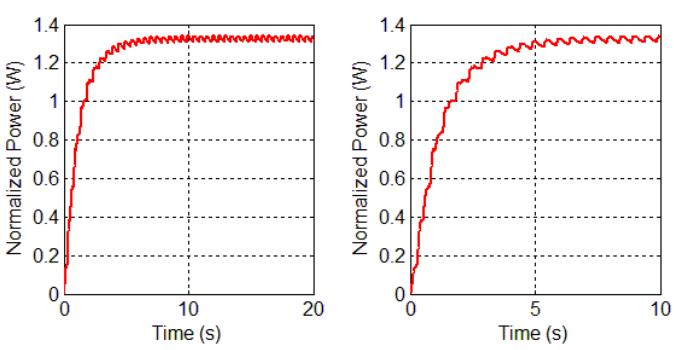

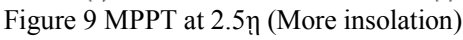
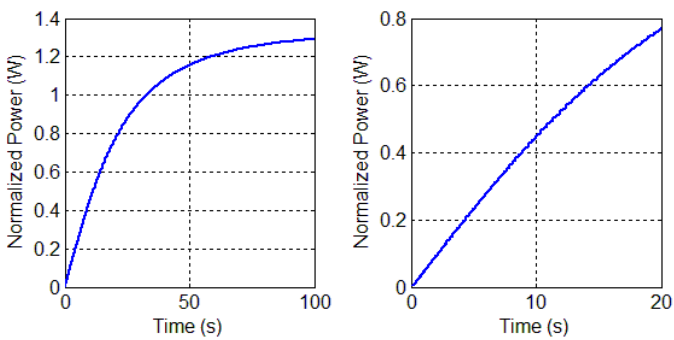

Figure 10 MPPT at $0.125 \eta$ (Less insolation)

Figures.8, 9 and 10 demonstrate that the proposed algorithm can rapidly locate MPP with guaranteed stability under varying insolation conditions. It is also shown that the MPP is rapidly located with increased irradiance level as in Figures 8 and 9 compared to Fig.10. However, the performance of the algorithm at low insolation is still successfully locating the MPP regardless of the speed.

\section{B. Experimental Evaluation of Proposed MPPT}

Here, we detail the experimental part of the proposed method. An off-the-shelf 4-32V to 0.8-32V four-switch, buck boost non-inverting converter was incorporated into an indoor built-in-house solar simulator as illustrated in Fig.11. This matched the PV module internal impedance to any given load and adjusted the operating condition to reach the maximum 
power point. This converter controls current as well as voltage using a built-in potentiometer. The load $(1 \Omega)$ voltage was controlled by adjusting the duty cycle of the power converter using the voltage potentiometer control pot and setting the current at maximum using the current potentiometer pot.

An indoor built-in-house solar simulator was utilized where a 17800 lumen LED floodlight was employed to emulate the sun and a KD70SX-1P PV module comprised the DC power source. The LED light was controlled by a PC using LPC1768 Mbed microcontroller to set the intensity of the solar simulator to any given value within the LED floodlight capability ( $78 \mathrm{~W} / \mathrm{m} 2$ max., $\sim 0.08$ of 1 sun). The overall system functionality is summarized in Table I and the hardware prototype of the converter incorporated with the solar simulator is shown in Fig. 12.

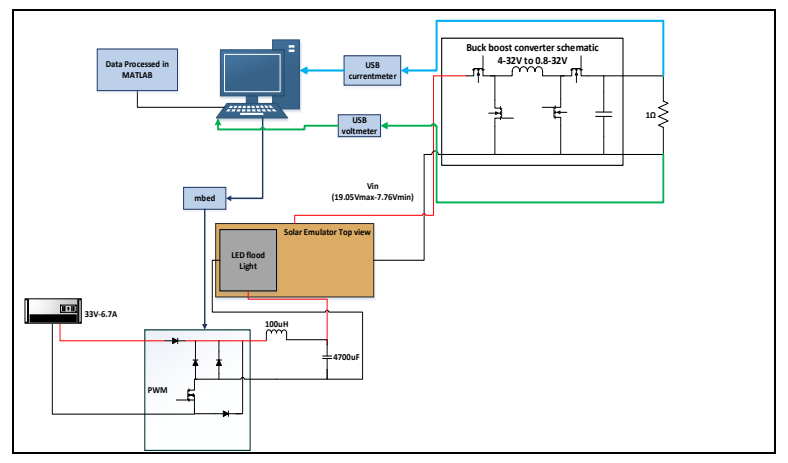

Figure 11 Microcontroller-based PV system with buck-boost converter incorporated
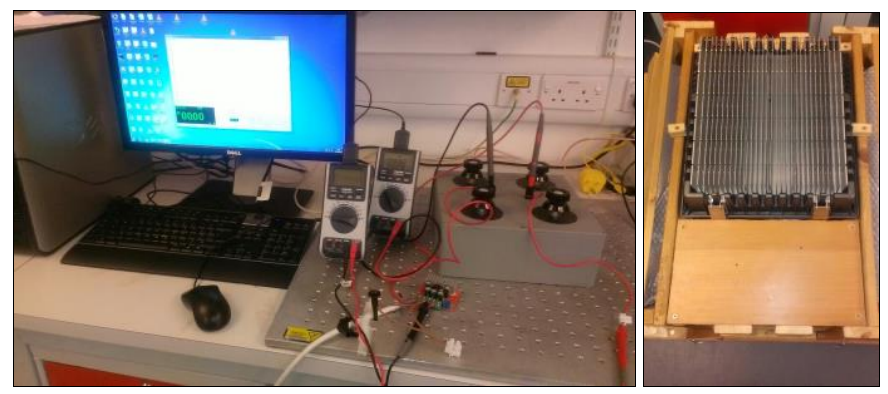

Figure 12 Hardware prototype

TABLE I. EleCtricAl PeRformance of PV System

\begin{tabular}{|c|c|}
\hline Short circuit current & $0.337 \mathrm{~A}$ \\
\hline Open circuit voltage & $19.8 \mathrm{~V}$ \\
\hline Maximum power & $4.3 \mathrm{~W}$ \\
\hline Amount of light intensity & $78 \mathrm{~W} / \mathrm{m}^{2}$ \\
\hline
\end{tabular}

The buck-boost converter was used in current mode by altering the duty cycle to maximize the current into the set load ( $1 \Omega$ resistive). The load current was observed using a USB digital multimeter and MATLAB provided data processing. The algorithm stopped tracking when maximum current and hence power, are reached. In the experiment, the converter is operated in buck mode with an overall maximum power from the solar simulator of approximately $4.3 \mathrm{~W}$. The single and multiple step algorithm was tested under three insolation conditions which were achieved by setting the LED current at maximum flux (maximum current 6.7A), 3A and $2 \mathrm{~A}$ as illustrated in Fig.13 and Fig.14 respectively. To highlight the algorithm, the forward steps after the MPP are illustrated in the curve.

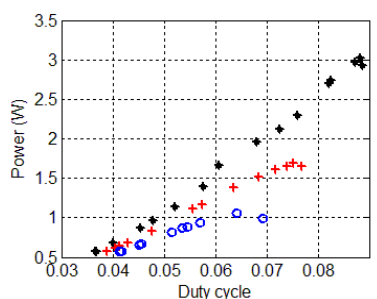

Figure 13 Single step forward-backward MPPT at $78 \mathrm{~W} / \mathrm{m}^{2}, 34.9 \mathrm{~W} / \mathrm{m}^{2}$ and $23.2 \mathrm{~W} / \mathrm{m}^{2}$

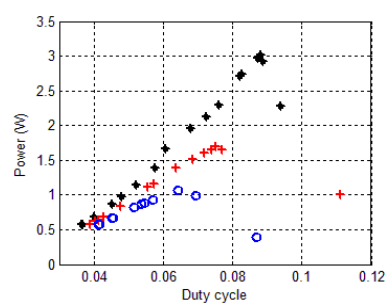

Figure 14 Multiple-step forward-backward MPPT at $78 \mathrm{~W} / \mathrm{m}^{2}, 34.9 \mathrm{~W} / \mathrm{m}^{2}$ and $23.2 \mathrm{~W} / \mathrm{m}^{2}$

It is obvious from Figure 13 and 14 that the proposed algorithm is successfully locating the MPP at $3.1 \mathrm{~W}$. Approximately $1.2 \mathrm{~W}$ is being consumed by the buck-boost converter.

The algorithm is further validated by testing the position of the point on the curve (up-hill and down-hill) to ensure that the MPP does not get lost with the change in insolation level as depicted in Fig. 15.
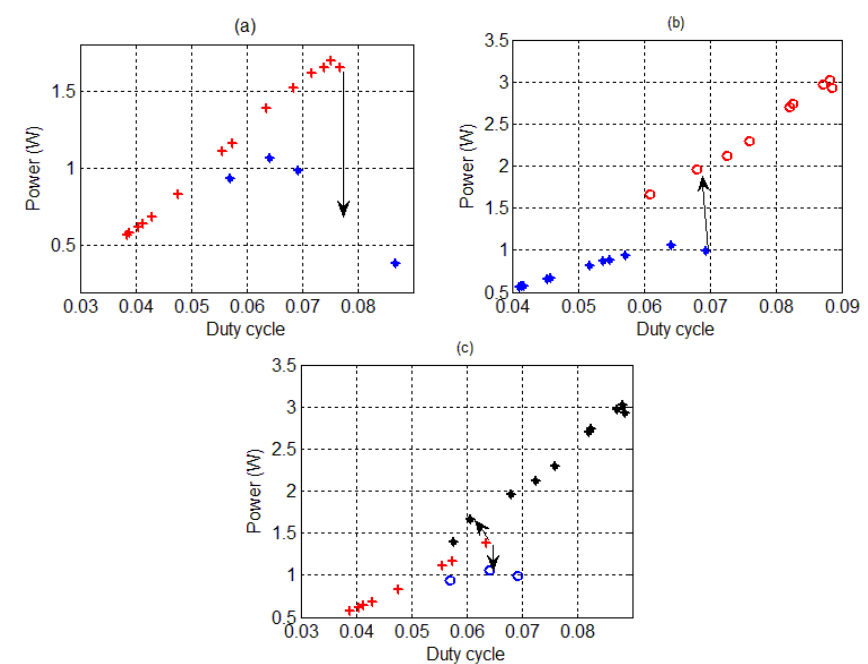

Figure 15 MPPT validation, a) downhill position $\left(34.9 \mathrm{~W} / \mathrm{m}^{2}\right)$ with sudden lower insolation $\left(23.2 \mathrm{~W} / \mathrm{m}^{2}\right)$, b) downhill $\left(34.9 \mathrm{~W} / \mathrm{m}^{2}\right)$ with sudden higher insolation $\left(78 \mathrm{~W} / \mathrm{m}^{2}\right)$, c) uphill position $\left(34.9 \mathrm{~W} / \mathrm{m}^{2}\right)$ with sudden higher

$\left(78 \mathrm{~W} / \mathrm{m}^{2}\right)$, and lower $\left(23.2 \mathrm{~W} / \mathrm{m}^{2}\right)$ insolation 
Figure 15 gives strong evidence that the proposed MPPT system successfully finds the MPP at rapidly varying irradiance conditions. Fig.15 (a) shows that point positioned on the downhill curve of $34.9 \mathrm{~W} / \mathrm{m}^{2}$ finds the optimum point with a sudden change in irradiance level $\left(23.2 \mathrm{~W} / \mathrm{m}^{2}\right)$ by checking forward power and backward power.

\section{CONCLUSIONS}

This paper proposed an algorithm and control technique of MPPT, which can maximize the power into any given load and accurately locate the position of MPP. Instead of P\&O method of numerical differentiation at PV side, we have proposed a single and multiple-step forward-backward difference optimizer which successfully tracks the position of MPP at various irradiance conditions and maximizes the power into any given load. This paper also shows a new heuristic PV model which is utilized in MATLAB/Simulink MPPT simulation to approximate the P-R curve. Experimental results for current mode MPPT at various insolation conditions validated the simulations.

\section{REFERENCES}

[1] M. de Brito, L. Galotto, L. Sampaio, G. de Azevedo e Melo, and C. Canesin, "Evaluation of the main MPPT techniques for photovoltaic applications," IEEE Trans. Ind. Electron., vol. 60, no. 3, pp. 1156-1167, Mar. 2013.

[2] B. Subudhi and R. Pradhan, "A comparative study on maximum power point tracking techniques for photovoltaic power systems," IEEE Trans. Sustain. Energy, vol. 4, no. 1, pp. 89-98, Jan. 2013.

[3] C. S. Chiu, "T-S fuzzy maximum power point tracking control of solar power generation systems," IEEE Trans. Energy Convers., vol. 25, no. 4, pp. 1123-1132, Dec. 2010.
[4] R. Ramprava and B. L. Mathur, "Intelligent controller based maximum power point tracking for solar PV system," Int. J. Comp. Appl., vol. 12, no. 10, pp. 37-41, 2011

[5] B. Khiari, A. Sellami, and R. Andoulsi, "MPPT control of photovoltaic pumping system based on discrete sliding mode," in Proc. Int. Renew. Energy Congr., Sousse, Tunisia, Nov. 5-7, 2010.

[6] H. Koizumi and K. Kurokawa, "A novel maximum power point tracking method for PV module integrated converter," in Power Electrons Specialists, 2005. PESC 2005-36th Annual Conference on IEEE, 2005, pp. 2081-2086.

[7] M. A. S. Masoum, H. Dehbonei, and E. F. Fuchs, "Theoretical and experimental analyses of photovoltaic systems with voltage- and current-based maximum power-point tracking," IEEE Trans. Energy Convers., vol. 17, no. 4, pp. 514-522, Dec. 2002.

[8] T. Esram, and P. L. Chapman, "Comparison of photovoltaic array maximum power point tracking techniques," IEEE Trans. Energy Convers., vol. 22, no. 2, pp. 439-449, Jun. 2007.

[9] F. Lui, Y. Kang , Y. Zhang and S. Duan, "Comparison of P\&O and Hill Climbing MPPT Methods for Grid-Connected PV Converter" in Industerial Electronics and Applications,2008, ICIEA 2008. $3^{\text {rd }}$ IEEE Conference on, 2008, pp. 804-807.

[10] N. Khaehintung, K. Pramotung, B. Tuvirat, and P. Sirisuk, "RISCmicrocontroller built-in fuzzy logic controller of maximum power point tracking for solar-powered light-flasher applications" in Industrial Electronics Society, 2004. IECON 2004. 30th Annual Conference of IEEE,2004, pp. 2673-2678.

[11] L. Elobaid, A. Abdelsalam, and E. Zakzouk, "Artificial neural network based maximum power point tracking technique for PV systems," in IECON 2012-38 $8^{\text {th }}$ Annual Conference on IEEE Industrial Electronics Society,2012, pp. 937-942.

[12] W. Xiao, W. G. Dunford, and A. Capel, "Application of centered differentiation and steepest descent to maximum power point tracking," IEEE Trans. Ind. Electron., vol. 54, no. 5, pp. 2539-2549, Oct. 2007.

[13] M. Hassani, S. Mekhilef, A.P. Hu, and N. R. Watson. "A novel MPPT algorithm for load protection based on output sensing control." In Power Electronics and Drive Systems (PEDS), 2011 IEEE Ninth International Conference on, 2011, pp. 1120-1124. 